\title{
Why care about kids?
}

\section{Ruth E Malone}

It is hard to argue against trying to protect kids from toxic exposures, but in the case of tobacco at least, the burden of doing so should not just be the job of parents and families. Rather, it should be shared by all of us through instituting better policies that protect everyone from exposure to secondhand smoke (SHS) and make tobacco products less accessible, less affordable and (perhaps most important) less attractive. In this special e-issue, a set of papers focused on children and youth shows the special challenges of doing so.

Far too many children are still involuntarily exposed to SHS in their homes. Mbulo et al, ${ }^{1}$ analysing data from the Global Adult Tobacco Survey for 21 countries, estimate that more than 500 million children under the age of 15 are exposed, including a shockingly high 79\% of Indonesian children. Further, too many of these children come from impoverished communities, compounding the inequalities they already face and showing why tobacco is a global social justice issue that worsens both health and socioeconomic disadvantage, as Hajizadeh and Nandi discuss. $^{2}$ In their study, poor children in almost all of the 26 countries studied were exposed to SHS daily. This exposure (including both prenatal and postnatal) comes at a cost: increased respiratory morbidity for children, as Snodgrass et al show in a study from Singapore.

However, we do know how to address part of the problem, at least in public settings. Smoke-free policies have been shown to have numerous beneficial effects for children. Lee et al report in this issue that smoke-free legislation in Hong Kong was associated with a significant reduction in lower respiratory infection-related hospitalisations for children, after controlling for the effects of outdoor pollution. ${ }^{4}$ In another important study, this time from Switzerland, smoke-free policies were associated with a reduction in preterm births, with greater reductions in areas with more comprehensive smoking bans and for pregnancies with longer gestational times under smoking bans. ${ }^{5}$

Against this backdrop, however, and despite their frequently professed claims

Correspondence to Professor Ruth E Malone, Department of Social and Behavioral Sciences University of California, San Francisco, CA 94118, USA; ruth.malone@ucsf.edu that they do not want kids to smoke, tobacco companies continue aggressive development and marketing of novel products that are attractive to youth. Abad-Vivero et al assess the impact of flavour capsules, a cigarette product innovation. ${ }^{6}$ In surveys with Mexican middle school students, flavour capsule variants of established brands were recalled and perceived as attractive, increasing interest in trying the products. The dynamic environment around e-cigarettes is also an important area of work. In an experimental study, Vasiljevic et al examined the relationship between viewing advertising of candy-like e-cigarette flavours and appeal of tobacco smoking in children 11-16 years of age. ${ }^{7}$ The study suggested that viewing flavoured e-cigarette advertisements did not increase the appeal of tobacco smoking, but did (perhaps unsurprisingly) increase interest in buying and trying e-cigarettes, with the flavoured e-cigarette advertisements increasing interest more than unflavoured e-cigarette advertisements.

The novelty of waterpipe has become another area for concern. Worldwide, there are reports of more youth trying or regularly using waterpipe (hookah, narghile, sheesha), often believing that it is less addicting and less toxic than cigarettes. A study of eighth and ninth graders from Lebanon ${ }^{8}$ demonstrates that even at low levels of consumption and frequency of use, youth may develop symptoms of nicotine dependence and experience craving. The authors call attention to social context as a key factor in shaping this new avenue for nicotine dependence.

But social context also applies to the world of tobacco products retailing, where marketing works the way marketing has always worked to influence kids to use tobacco. Robertson et al ${ }^{9}$ conducted a meta-analysis of studies examining point-of-sale (POS) tobacco promotion and smoking outcomes in youth and found that children and youth who were more frequently exposed to POS tobacco promotions had 1.6 times higher odds of having tried smoking, as well as higher odds of being susceptible to smoke in the future. Banning POS tobacco promotion, which is not needed to reach existing smokers, is an obvious policy remedy.

Tobacco outlet density is another area of great current interest as jurisdictions begin to wrestle with the ubiquitous presence of tobacco and its links to youth smoking, as well as relapse among smokers trying to quit. A study from New Zealand $^{10}$ found mixed results: higher retailer density around schools was not associated with current smoking, but students attending schools with high tobacco retailer density were more susceptible to smoking and more likely to try and purchase tobacco than students in zero density areas.

In a US study examining results of 2015 Food and Drug Administration (FDA) inspections of tobacco retailers, ${ }^{11}$ retailers in areas with higher populations of poor and minority residents were more likely to fail an underage tobacco buy inspection. That is, simply growing up in a neighbourhood with a greater proportion of American Indian, black or Latino residents was associated with a greater likelihood that kids could buy tobacco from retailers in violation of existing law. Place matters: the social justice implications of the community environment are an important topic for future work.

Finally, two papers explore aspects of how adolescents perceive tobacco control measures. In an experimental study of more than 1000 adolescent smokers, Andrews et $a l^{12}$ examine the effects of graphic warnings and 'plain' packaging, adding to a growing body of evidence demonstrating that both of these packagefocused measures further the goal of discouraging youth smoking. A qualitative study from Singapore ${ }^{13}$ captures the perspectives of youth themselves about antismoking campaigns. Messages with autonomy-supporting language were more persuasive, whereas fear appeals were met with defensive responses.

More than 20 years ago, Dr David Kessler, then head of the US FDA, called tobacco use a 'paediatric disease', arguing that few individuals over age 19 start smoking. ${ }^{14}$ While in the face of increased industry target marketing to young adults this may not still be as true as it once was, finding a way to end youth tobacco uptake of and addiction to tobacco products must be a priority. However, too often policymakers' response is that we must 'increase education' about the harms of tobacco use, especially in schools. But while non-controversial, the evidence of effectiveness for this approach is mixed.

Instead, the most effective youth campaigns have had broader social engagement and focused on exactly what the Singaporean study suggested: increasing youth autonomy. For example, the strong and effective 'Truth' campaigns like the 
one featured on this issue's cover have helped youth see their important role in battling Big Tobacco and ending this industrially produced pandemic. Can this generation 'finish it'? ${ }^{15}$ Only time will tell, but creating policy environments that make it easier for them to do just that may help.

Competing interests None declared.

Provenance and peer review Not commissioned; internally peer reviewed.

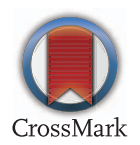

To cite Malone RE. Tob Control 2016;25:e69-e70.

Accepted 23 November 2016

Tob Control 2016;25:e69-e70.

doi:10.1136/tobaccocontrol-2016-053555

\section{REFERENCES}

1 Mbulo L, Palipudi KM, Andes L, et al. Secondhand smoke exposure at home among one billion children in 21 countries: findings from the Global Adult Tobacco Survey (GATS). Tob Control 2016;25: e95-100.

2 Hajizadeh M, Nandi A. The socioeconomic gradient of secondhand smoke exposure in children: evidence from 26 low-income and middle-income countries. Tob Control 2016;25:e146-55.

3 Snodgrass AM, Tan PT, Soh SE, et al. Tobacco smoke exposure and respiratory morbidity in young children. Tob Control 2016;25:e75-82.

4 Lee SL, Wong WHS, Lau YL. Smoke-free legislation reduces hospital admissions for childhood lower respiratory tract infection. Tob Control 2016;25: e90-3.

5 Vicedo-Cabrera AM, Schindler C, Radovanovic D, et al. Benefits of smoking bans on preterm and early-term births: a natural experimental design in Switzerland. Tob Control 2016;25:e135-41.

6 Abad-Vivero EN, Thrasher JF, Arillo-Santillán E, et al. Recall, appeal and willingness to try cigarettes with flavour capsules: assessing the impact of a tobacco product innovation among early adolescents. Tob Control 2016;25:e113-19.

7 Vasiljevic M, Petrescu DC, Marteau TM. Impact of advertisements promoting candy-like flavoured e-cigarettes on appeal of tobacco smoking among children: an experimental study. Tob Control 2016:25:e107-12.
8 Bahelah R, DiFranza JR, Fouad FM, et al. Early symptoms of nicotine dependence among adolescent waterpipe smokers. Tob Control 2016;25: e127-34.

9 Robertson L, Cameron C, McGee R, et al. Point-of-sale tobacco promotion and youth smoking: a meta-analysis. Tob Control 2016;25:e83-9.

10 Marsh L, Ajmal A, McGee $R$, et al. Tobacco retail outlet density and risk of youth smoking in New Zealand. Tob Control 2016;25:e71-4.

11 Vasiljevic M, Petrescu DC, Marteau TM. Impact of advertisements promoting candy-like flavoured e-cigarettes on appeal of tobacco smoking among children: an experimental study. Tob Control 2016;25:e107-12

12 Andrews JC, Netemeyer RG, Burton S, et al. Effects of plain package branding and graphic health warnings on adolescent smokers in the USA, Spain and France. Tob Control 2016;25: e120-6.

13 Shahwan S, Fauziana R, Satghare P, et al. Qualitative study of Singaporean youths' perception of antismoking campaigns: what works and what does not. Tob Control 2016;25:e101-6.

14 Hilts PJ. F.D.A. Head calls smoking a pediatric disease. 1995. http://www.nytimes.com/1995/03/09/ us/fda-head-calls-smoking-a-pediatric-disease.html

15 The Truth. http://www.thetruth.com/about-truth 